\title{
Negotiating Masculine Spaces: Attitudes and Strategies of First-Year Women in Engineering
}

\section{Dr. Marie C Paretti, Virginia Tech}

Marie C. Paretti is an Associate Professor of Engineering Education at Virginia Tech, where she co-directs the Virginia Tech Engineering Communications Center (VTECC). Her research focuses on communication and teamwork in engineering, design education, and engineering identity. She was awarded a CAREER grant from NSF to study expert teaching practices in capstone design courses nationwide, and is co-PI on NSF . Her work includes studies on the teaching and learning of communication, the effects of curriculum on design cognition, the effects of differing design pedagogies on retention and motivation, the dynamics of cross-disciplinary collaboration in both academic and industry design environments, and gender and identity in engineering.

Ms. Courtney S Smith, 


\title{
Negotiating Masculine Spaces: Attitudes and Strategies of First- Year Women in Engineering
}

\begin{abstract}
Research throughout the late $20^{\text {th }}$ and early $21^{\text {st }}$ century explored the kinds of barriers and challenges women faced in entering the male-dominated space of engineering. But since work such as Tonso's landmark study, On the Outskirts of Engineering ${ }^{1}$, a number of cultural changes have occurred, and expectations for what women can do and what fields they can enter. Have those shifts made a difference for women entering engineering programs? In this paper, we explore that question through interviews with 10 women selected based on the results of a survey of first-year engineering students in a large general engineering program at a mid-Atlantic university. Interview subjects were selected based on high domain and high gender identification; that is, the 10 women who were interviewed perceived both being female and being an engineering as important to their self-concept. The interviews were transcribed and analyzed using open-coding procedures to identify ways women experience the relationship between their gender and their engineering identities, their experiences with male students (e.g. on design teams, studying for exams, completing homework assignments), and their strategies for navigating gendered dynamics such as peers who did not value their input or who expected them to take on certain roles. The participants rarely responded directly to questions about perceived gendered experiences, but when talking about their experiences in classes or on teams, did describe ways in which they had negotiated their space and their identity, earning respect or demonstrating competence to gain a voice at the table. At the same time, these participants saw no need to give up or mask their femininity, and spoke about embracing and moving easily between identities. The findings suggest that while overt experiences of gender stereotypes or sexism may be decreasing, biases persist; at the same time, women entering engineering programs are both aware of and have strategies for negotiating those biases. The findings suggest opportunities for educators to strengthen women's self-concepts as both women and engineers.
\end{abstract}

\section{Introduction}

It has been more than 10 years since Tonso conducted her ground-breaking ethnographic study of engineering design teams that explored the highly gendered nature of team interactions and the kinds of challenges women face in such environments ${ }^{1}$. Studies of the experiences of women engineers by Faulkner ${ }^{2,3}$, Dryburgh ${ }^{4}$, and others have explored the gendered dynamics of engineering work, the divisions between being “feminine” and being "an engineer," and the impact of such divisions on engineering identity. And gender gaps persist not only in engineering but across a range of fields; Anne-Marie Slaughter's summer 2012 article in The Atlantic is one in an ongoing series of articles that describe the challenges women face as professionals in the contemporary workplace ${ }^{5}$.

Yet certain cultural changes have occurred, and expectations for what women can do and what fields they can enter have shifted since Tonso’s fieldwork. Contemporary studies within engineering education report mixed results with respect to women’s experiences. In a 2001 study, Besterfield-Sacre et al. found incoming male students rated themselves higher in terms of basic knowledge, problem-solving, and engineering abilities ${ }^{6}$, and a 2003 study by Bell et al. found that stereotype threat had negative consequences on test performance for women 
engineering students ${ }^{7}$. Wolfe and Powell also found significant biases against feminine speech patterns in design teams ${ }^{8}$. But in a 2005 study, Marra and Bogue report differences in selfefficacy between male and female engineering students only for math, where women reported higher scores ${ }^{9}$, confirming findings of other studies associated with self-efficacy. In 2010, Jones et al. found mixed results in a study of first-year engineering students, with no statistically significant differences associated with attainment, interest, utility, identification, or career-plans; although men did score higher with respect to self-efficacy and expectancy, the difference was significant only for expectancy ${ }^{10}$.

In a related study, Jones et al. found few differences between men and women with respect to engineering identification, gender identification, GPA, or likelihood of changing major, and women reported higher perceptions of engineering ability ${ }^{11}$. The analysis also indicated no effects for stereotype threat; notably, however, although both men and women scored relatively low with respect to endorsing negative stereotypes about women in engineering, men did report more endorsement (3.4 for men vs. 2.4 for women, with 7 representing strong endorsement) ${ }^{11}$.

The shifting and often conflicting patterns with respect to women's experiences in engineering raise questions about how the climate has or has not changed, what strategies and structures women are leveraging to negotiate engineering culture, and what practices institutions should be employing to continue to increase gender diversity in the field. Toward these ends, this paper presents a qualitative study undertaking in conjunction with Jones et al.'s quantitative analyses ${ }^{10}$, 11 to better understand women's experiences in the contemporary engineering climate. Specifically, we address two questions:

1. Do women in engineering still experience negative stereotyping, and if so, how do those stereotypes manifest themselves?

2. How do women negotiate engineering culture in general and negative stereotypes in general in the current climate?

Note that although the 2013 study does indicate the continued presence of negative stereotypes, albeit at low levels, the lack of measurable effect on women's performance made it important to understand if and how women experienced those stereotypes before exploring how they negotiate engineering culture.

\section{Methods}

This study employed a phenomenological approach ${ }^{14}$ to understand the experiences of women who are highly identified with their gender and with engineering. Women were interviewed at the end of their first year in engineering, after they completed a two-semester introduction to engineering sequence, along with related courses in math, sciences, and humanities. These qualitative interviews were part of a larger mixed methods study exploring identity, stereotype threat, motivation, retention, and persistence in first-year engineering students ${ }^{10,11}$. Interview participants were selected based on the results of a survey of first-year engineering students in a large general engineering program at a mid-Atlantic university. 
Sample

Because stereotypes typically pose the greatest threat for individuals who are highly identified with the domains in question ${ }^{12,13}$, this study focused on women who scored highly on both engineering identification and gender identification - that is, perceived both being female and being an engineering as important to their self-concept (for a full discussion of the scales, see Jones et al. ${ }^{10,11}$ ). Women who scored highly on both scales were invited to participate in interviews at the end of their first-year. Ten women responded and interviews were conducted by the first author; the sample size is consistent with phenomenological methods ${ }^{14}$. Note, however, that because of scheduling issues, six of the interviews were conducted in the spring, at the conclusion of the women's first year, while an additional four were conducted in the fall, at the beginning of their sophomore year. The interviews were audio-recorded and transcribed verbatim for analysis; in addition, the interviewer kept field notes for all interviews. All data collection was conducted with the approval of Virginia Tech’s Institutional Review Board (IRB 07-403).

\section{Protocol}

A semi-structured interview protocol was developed to more fully explore a range of issues associated with participants' experiences in engineering, including their choice of major, their experiences during the first year, their identification with engineering, and their identification with gender. The complete protocol is included in Appendix A.

\section{Analysis}

Consistent with phenomenological approaches ${ }^{14}$, the interviews were transcribed and analyzed using open-coding procedures to identify ways women experience the relationship between their gender and their engineering identities, their experiences with male students (e.g. on design teams, studying for exams, completing homework assignments), and their strategies for navigating gendered dynamics such as peers who did not value their input or who expected them to take on certain roles. The two authors read through the interviews independently to identify key themes, and then met to discuss those themes and develop a preliminary code book. The authors then applied the code-book independently to one of the 10 interviews; this process yielded high agreement on the codes, and the remaining interviews were coded accordingly, with half coded by the first author and half coded by the second author.

\section{Results}

\section{Overview}

Collectively, the interviews revealed the low but consistent presence of stereotypes regarding women in engineering, but also indicated that overall these stereotypes appeared to carry little weight as the women described them. At the same time, these participants saw no need to give up or mask their femininity, and several spoke about embracing and moving easily between identities. As they negotiated engineering culture, three key strategies emerged consistently across the interviews: 
- Hard work: overall, the interview participants expressed high levels of confidence about their work and believed that their work would easily stand on its own to dispel any concerns their male colleagues might hold.

- Structures Support Systems: Most of the participants interviewed participated in and explicitly leveraged a range of formal support structures provided by the university, including pre-engineering summer programs, residential learning communities, and engineering Greek organizations oriented toward service and/or scholarship.

- Informal Support Systems: In addition to the structured support systems, most participants also reported informal support systems. In some cases, these support systems were based on prior relationships - friends or family members already at the university; in other cases, they emerged from the structured support systems (e.g. friends made through a learning community that became study partners).

The following sections provide more detail about participants' experiences with gender stereotypes, gender identity, and the three strategies noted above. Quotations are identified by participant ID number.

\section{Gender Stereotypes}

The participants rarely responded directly to questions about perceived gendered experiences, but when talking about their experiences in classes or on teams, did describe a variety of experiences that expressed subtle forms of stereotyping.

In some cases, the stereotypes were explicitly present, as in the case where a participant described the reaction of a high school teacher to her choice of engineering:

...he was, like, no way. I can never see you doing that, and I took that as an insult. Like, it hurt my feelings because ... he's, like, I picture you going into fashion design or something like that. [92208_430]

In other cases, biases manifest themselves in subtle ways as women find themselves working with men who don't seem to quite trust them or have confidence in their abilities, but don't make overt comments:

I just got a lot of felling a lot of times, like, I did a good bit of that project, and I feel like a lot of times the stuff I did was, like, second guessed, like a lot of them went back and checked it. There was one guy in the group particularly that never took any of my ideas.....[In another group] we had to come up with a list of solutions ... and he just refused to write anything [I said] down, and it didn't bother me until the kind next to me repeated what I said and he wrote it down. [92508_330]

Such instances, however, seem the exception rather than the rule in these women's experiences, but most had either experienced it once or knew someone who had. More common are experiences in which the stereotypes are invoked, but ostensibly undercut with humor: 
... on the teams, the guys would make stupid comments about girls ... Just joking around, just being like, you know, we'll do the hard stuff and you guys [i.e. the girls on the team] can just type it up, and we'll be like yeah, okay, like we haven't done the whole project.... I ignore the comments and just kind of laugh along with them and roll my eyes, but it's, yeah, it's strange... [43008_330]

In each case where participants mentioned explicit comments, they were always in the context of a "joke" - an intentional invocation and playing up of the stereotype, coupled with the participants own laughter about the "stupidity" of such comments. Nonetheless, the persistence of such jokes, along with the more subtle as well as the more explicit forms of bias, remind us that even as perceptions changed significantly in recent years, it has not changed completely.

Finally, stereotypes about women in engineering also emerge through reminders from those outside the field; several participants noted that other people often reacted to the participants' choice of major with questions about what it was like to be a minority in a field, creating a kind of low-level reminder of the space that women continue to occupy. As one participant put it,

...a lot of people ask you, oh, what's it like being the only girl in your class? And then you start to think, yeah, yeah, like I really didn't notice that. [92208_430]

What is striking across all of these comments, however, is the degree to which the participants repeatedly dismiss the stereotypes; the presence of stereotypes becomes something interesting to observe, and occasionally annoying, but rarely overwhelming or threatening.

\section{Gender Identification}

As noted in the description of the interview participants, the women invited to participate in these interviews also scored highly on the construct of gender identification as measured via survey. That is, they all reported that being a woman was an important component of their selfconcept. This gender identification emerged across the interviews as well, as participants described the ways in which aspects of their femininity or gender remained important to them, though the participants had a range of ways of describing their gender identities, as the following examples suggest:

I'm very feminine....I am very feminine and, like, I like going to the movies with the girls and, like, shopping and all of that. [92208_430]

I'm definitely more girly-girl than a tomboy I guess. I don't know and like for work I'll get nice and dressed up and put on makeup [43008_330]

I've always kind of like being the one girl in a group of guys ... like make me feel special or whatever. I’ve just been used to it most of the time [50108_200]

Importantly, these participants, whether they saw themselves as highly feminine or as the tomboy who likes to hang with guys, rarely if ever considered their gender something to hide or to erase. As one participant explained, 
if I'm hanging out with, like, some guy friends who are all engineering and they call me dude or something, I'm, like, wait, don't call me dude, like, they'll treat you as one of the guys sometimes. [92208_430]

Gender is clearly a component of their identies, and one they are both aware of and comfortable with as they move through an engineering curriculum.

\section{Hard Work}

In many cases, the women responded to both implicit and explicit bias through sheer hard work; simply by being good at what they do, they believe they can overcome the negative or stereotypes or the lack of trust they experience from others. This reliance on personal ability and effort is clear, for example, in one woman's description of her group project experience:

I felt like at first, like, [the guys on the team] were overpowering with their ideas and they all knew what solar power was, and I was, like, what is this? [laughter] But I mean, I'm sure in this other group there could be a guy who didn't know what was going on.... But it wasn't the fact that I'm a girl that I didn't know what solar power was, but I looked it up and read about it and then I, I caught up to speed with the group, and, uhm, it went well, our project. [92208_430]

Another participant explained more generally,

...if they see, like, you kind of belong there, like, oh, well, they're actually working, they are dedicated towards this kind of stuff, they are going to stick with then; they are not going to care whether or not you're a girl or a guy or anything like that. [50108_200]

In cases like those above, working hard is simply part of being a competent and engineer. It is, at least on the surface, disconnected from gender; both men and women, the participants imply, have to work hard to prove themselves. It becomes, as one participant notes, part of what all engineers do:

Everyone makes fun of me because I literally sit in my room and do work all day. Every time they come in there's one of the kids down the hall always makes fun of me because he says, "Every time I come in you're doing math. No matter what, you're always doing math every time I come in." I say, "Yeah, that's pretty much [how it is]. I'm always doing something. [43008_1015]

But in other cases, this emphasis on hard work is clearly about disproving stereotypes:

I feel that I need more than being a good student, I need to show that I'm doing this, I need to be given credit for this because first of all it takes a lot of guts to go into something, into a class, which is all guys, it takes guts to approach them and ask them how did they do this? It takes guts to study a tough course ... [91808_945]

Similarly, another participant described her experience in a group directly in terms of shattering the stereotype: 
A lot of times it's kind of difficult because I felt, it was funny to me because a lot of people think, oh, girls in engineering. They're not going to know what they, they are not going to know what to do. They are going to be like fish out of water, and I was actually the driving force behind those of my projects. As in I was the one who was, like, okay, we're going to meet here, we're going to do this, this is what we need to get done. I was the one who had to tell everyone to, like, you know, tunnel vision. Stay on track, do it. [43008_1015]

Almost universally, the participants in this study had a clear commitment to working hard at their courses to achieve success.

\section{Structured Support Systems}

Hard work, however, was never treated as a panacea or as the only approach, and the women participating in these interviews all described multiple support systems that provide both technical support (help on homework, help understanding difficult concepts) and social support (feeling connected, having friends inside or outside engineering to do things with). In many cases, these support systems are linked to structured elements of the university such as residential learning communities or pre-college programs. One participant, for example, explained the benefits of a residential learning community for engineering women:

It was a really good experience because not knowing anyone coming here it was nice on my hall there were one hundred girls and there was at least, like, five or six of them in every class so I knew people. And we all had the same homework and we had all, like, had study groups together. [Interviewer: So you did a lot of studying with them?] Uh-huh, yeah, because you would always see everyone in the lounge with their books open and you'd be like, uh-oh, am I supposed to be doing that now? [laughter] Then you would go in and join them. It was really helpful. [92208_430]

Notably, however, the participants cited a broad range of structured experiences rather than a single dominant factor. In some cases, women participated in residential learning communities outside engineering (e.g. in leadership). Other participants described the value of Greek organizations, particularly ones with professional components:

It's so great. It's a professional sorority.... But it's also a social sorority so you kind of get the best of both worlds there because they have professional weekends, they have etiquette classes, they have resume building workshops. So they do all kinds of things that really help professionally and you have your alumni connections to actual firms and businesses and, like, that's really helpful, too, because, you know, you can talk to them about what they do and maybe try to get jobs with them because they can be like, oh, well, this is my sister.[050208_1100]

For others, the support came from summer programs designed to prepare students for their first year in engineering:

...what they did is they were like you're going to be an engineer, it's going to be hard, so we're going to help you transition from high school into college, and I went there and I had a bit of misgivings because I thought, oh, I'm going to spend my summer doing, like, coursework and stuff, but it was actually it was a lot of fun -- it was a lot of work, too -- but it 
was a lot of fun, and I really enjoyed it and it kind of helped me solidify the idea that this is what I really wanted to do. [43008_200]

The value of these support structures, coupled with the broad range of structures these women accessed, clearly provides qualitative support for the role such programs play in helping those in underrepresented groups build the networks needed to support them academically and professionally.

\section{Informal Support Systems}

In most cases, however, even when structured support systems are presented, they are coupled with (and often lead to) informal networks of support. A number of interview participants described scenarios in which structured systems led to close informal working relationships, as the examples below indicate:

I'm also good friends with a lot of people from [the summer program], who also happen to be in Dorm X, which is the all-guy engineering program. So, a lot of the times what happens is if I know I have a lot of work especially in engineering related topics, I just go over to Dorm $\mathrm{X}$ and start working in the study lounge and find a buddy. [43008_200]

They all my best friends, like, six girls from [the female engineering residential learning community]. My roommate this year was on my floor last year, too. [92208_430]

In other cases, informal support networks grew from prior relationships; many of the women interviewed had friends or family members attending the university and studying engineering, and these connections formed the basis for important learning support systems. One woman explains her network as follows:

So, there's a lot of people from [my hometown] here so like we have a little network of [hometown] people, and one of my good friends is an engineering, and he live in Dorm X and so he goes, since he's a boy in engineering, he knows a lot more guys in engineering ... [43008_330].

Later, she also describes family connections that provide motivation and help her contextualize courses that otherwise seem meaningless:

...my sister is older than me, of course, so she has older friends and like I've talked to them once I got in engineering and they're like you really do need to know that stuff. [430008_330]

Another participant describes the role of an older sibling in similar ways:

It helps that I have some, well, my brother and his friends, a couple of them are engineers, too....So, I hear their experiences. My boyfriend is a senior in civil engineering so he kind of talks to me about stuff, uhm, and his friends, a lot of his friends, are in the building construction program so I've heard stuff from them so it helps having a lot of older connections at the school [50208_1100] 
These informal networks provide the interview participants with access to help on homework, experiences of older students that inform decision making, guidance on the relative importance of various courses and topics, and a host of related support issues.

\section{Discussion and Conclusions}

The findings from these interviews suggest that while overt experiences of gender stereotypes or sexism may be decreasing, some biases persist. Participants in this study demonstrated some of the same reluctance to talk about these gender biases seen in the work of earlier scholars such as Tonso $^{1}$ and Dryburgh ${ }^{4}$. At the same time, the women who participated in these interviews all enacted multiple strategies for negotiating those biases, some of which reflect shifts from earlier patterns. One component of that negotiation is rooted in self-reliance; these women demonstrated high levels of confidence in their ability, and were able to both work hard and identify resources (the internet, textbooks) that provided them with the necessary knowledge and skills. Access to internet sources, in particular, may represent a shift in the kinds resources women have readily available. Notably, these young women also leveraged personal and professional networks of friends, family, and faculty to provide support on homework assignments, group projects, and other course-related issues as well as to provide an effective social circle of support and affirmation. This access to supportive social networks may also represent a shift from earlier work, though it remains consistent with the findings from studies demonstrating the importance of networks and social capital.

In many cases, however, these informal networks strongly facilitated by existing campus structures that provided support networks. Residential learning communities, group projects in classes that created collaborative rather than competitive team dynamics (including assigning multiple women to a team and monitoring team roles), Greek and professional organizations associated with engineering, and similar structures all provided important avenues for these young women to negotiate the engineering curriculum broadly and the low-level (and occasionally blatant) biases that persist within the culture. These structures were particularly important for women who lacked pre-existing networks through friends and family, but even those who already knew engineering students at their university often coupled their informal networks with more formal structures.

The importance of these networks also represents a shift from earlier studies and suggest increasing opportunities for women to successfully negotiate biases and barriers. The importance of formal structures, as well as the diversity of structures leveraged by these interview participants, make it clear that even as the climate within engineering may be shifting, universities need to continue to provide structures that enable under-represented populations, including women, to build and access the kinds of informal networks more easily experienced by dominant populations. These structures may be even more important for students who enter engineering programs without ready access to informal networks supplied by family, friends, and high school classmates attending the same institution and pursuing the same major. Given the prominent role these informal networks play in supporting student success, universities have an ongoing responsibility to help women develop these structures in a field where, despite decades of work, they remain underrepresented. At the same time, the availability of these resources, and women's ability to draw on them, does not negate the need to continue to address the barriers themselves and continue to explore ways to shift the larger culture of engineering. 


\section{Acknowledgements}

This material is based upon work supported by a grant from Virginia Tech and by a grant from the National Science Foundation under Grant No. 0936704. Any opinions, findings, and conclusions or recommendations expressed in this material are those of the author(s) and do not necessarily reflect the views of the National Science Foundation. In addition, the authors thank those individuals who collaborated on the larger study from which this work was drawn: Serge Hein, Tamara Knott, and Brett Jones.

\section{Bibliography}

1. Tonso, K.L., On the Outskirts of Engineering: Learning Identity, Gender, and Power via Engineering Practice. 2007, Rotterdam: Sense Publishers.

2. Faulkner, W., "Dualisms, Hierarchies and Gender in Engineering." Social studies of science, 2000. 30 Part 5: p. 759-792.

3. $\quad$ Faulkner, W., "'Nuts and Bolts and People': Gender-Troubled Engineering Identities." Social studies of science, 2007. 37(3): p. 331-356.

4. Dryburgh, H., "Work Hard, Play Hard: Women and Professionalization in Engineering - Adapting to the Culture." Gender \& Society, 1999. 13(5): p. 664-682.

5. $\quad$ Slaughter, A.-M. "Why women still can't have it all." The Atlantic, 2012.

6. Besterfield-Sacre, M., et al., "Gender and Ethnicity Differences in Freshmen Engineering Student Attitudes: A Cross-Institutional Study*." Journal of Engineering Education, 2001. 90(4): p. 477-489.

7. Bell, A., et al., "Stereotype Threat and Women's Performance in Engineering." Journal of Engineering Education, 2003. 92(4): p. 307-312.

8. Wolfe, J. and E. Powell, "Biases in Interpersonal Communication: How Engineering Students Perceive Gender Typical Speech Acts in Teamwork." Journal of Engineering Education, 2009. 98(1): p. 5-16.

9. Marra, R. and B. Bogue, "Self Efficacy in Women Engineering Students: Three Years of Data at U.S. Institutions," in American Society for Engineering Education. 2007: Honolulu, HI. p. 15 pp.

10. Jones, B.D., et al., "An analysis of motivation constructs with first-year engineering students: Relationships among expectancies, values, achievement, and career plans." Journal of Engineering Education, 2010. 99(4): p. 319-336.

11. Jones, B.D., C. Ruff, and M.C. Paretti, "The Impact of Engineering Identification and Stereotypes on Undergraduate Women’s Achievement and Persistence in Engineering." under review.

12. Schmader, T., "Gender identification moderates stereotype threat effects on women's math performance." Journal of Experimental Social Psychology, 2001. 38: p. 194-201.

13. Schmader, T., M. Johns, and M. Barquissau, "The costs of accepting gender differences: The role of stereotype endorsement in women's experience in the math domain." Sex Roles, 2004. 50(11/12): p. 835850.

14. Patton, M. Q. (2002). Qualitative Research \& Evaluation Methods (3rd ed.). Thousand Oaks: Sage Publications.

\section{Appendix A: Interview Protocol}

\section{Enrolling in an Engineering Program}

1) Please describe for me your experience of making the decision to enroll in an engineering program.

2) At this point, how important is it to you to be an engineering student?

Engineering-Related Coursework and Relationships 
3) What is your opinion of the engineering-related courses that you've taken so far? This would include engineering courses and any math or science courses that you've taken.

4) How important is it to you to be interested in engineering-related courses?

4.5) How important is it to you that your engineering-related courses will be of use to you when you become an engineer?

5) Could you please describe for me how well you've done so far in your engineering-related courses.

6) Please tell me how important it is to you to do well in your engineering-related courses.

7) How important is it to you to be seen as a good engineering student by other engineering students?

Possible probes: instructors in your engineering-related courses, friends, family

8) Please describe for me how well you feel you fit in with other students in your engineering-related courses.

Possible probes: male students, female students, other students in your engineering program, other engineering students

9) How important is it to you to fit in with other students in your engineering-related courses?

Possible probes: male students, female students, other students in your engineering program, other engineering students

\section{Choice of Major and Career Goals}

10) Do you have any plans to change your major? If so, please describe them for me.

11) At this point, what kind of work do you see yourself doing after you graduate?

12) At this point, how important is it to you to have a career as an engineer after you graduate?

\section{Gender-Related Issues}

13) Please describe for me what it has been like for you, being a woman in your engineering program.

14) How much does being a woman contribute to how you see yourself as a person?

15) How important is being a woman to you, as far as how you are viewed by others?

Possible probes: other engineering students, instructors in engineering, friends, family

16) Is there anything else that you think would be important for me to know, as far as the topics that we've discussed today? 